\title{
The hippocampus, conditional operations, and cognition
}

\author{
RICHARD HIRSH \\ McGill University, Montreal, Quebec H3A IBI, Canada
}

\begin{abstract}
This paper argues that the hippocampus is involved in mnemonic processes by virtue of being necessary to the execution of conditional operations occurring during the retrieval of acquired information from memory. A series of experiments indicating that animals suffering from hippocampal dysfunction are incapable of behaving conditionally in learning situations is described. Other effects of hippocampal dysfunction are then explained in terms of disruption of conditional retrieval processes. The paper continues by defining retrieval as the selection of one piece of information from among many possible candidates. It is argued that such a process is of little utility in basic associative theories, as associative processes result in one predominant memory for any given stimulus. Cognitive theories, in contrast, have held that many things may be known about a given stimulus and thus selection among memories is mandatory. The process of conditional retrieval is then defined as the result of interaction between a categorical operator, representing global factors, and a local operator, representing local considerations. Interaction between these two types of operators is shown to be necessary for constructing dimensional representation, including maps. To hold that the hippocampus is involved in the construction of dimensional representations in general affords an opportunity to begin relating cognitive processes to physiological knowledge and to answer some of the broad conflicts between associative and cognitive theories of learning. It is further suggested that studies of hippocampal development may shed light on cognitive development.
\end{abstract}

Studies on the function of brain structures in producing behavior must be done within some sort of psychological framework; otherwise the results cannot be interpreted. The capability of the framework in interpreting the results is, in turn, an indicator of the validity of the framework. Thus, studies of the behavioral function of structures deal not only with neurological mechanisms, but also with psychological processes. Studies of the role of the hippocampus in producing behavior have something quite important to say about the nature of learning and memory and, very possibly, about cognitive development.

\section{Memory}

Most of us here presently hold that the hippocampus is involved in some forms of memory but not in others. The main inspiration for such theories still is that clinical findings indicate that hippocampal lesions are followed by pervasive anterograde and some retrograde amnesia (Penfield \& Milner, 1958), while nonmnemonic capacities are unaffected. There was some question as to whether such conclusions should be applied to hippocampal functions in animals, in that hippocampally ablated animals learn at normal rates in a wide variety of relatively simple learning paradigms. Deficits in these animals were not observed

The research described in this paper was supported in part by grants from the Natural Sciences and Engineering Research Council of Canada (No. A 7918) and the Ministry of Education for the Province of Quebec (No. EQ-84), to Dalbir Bindra. until modification of already established learning was required. The hippocampus was thus thought to be involved in processes only incidental to the formation and expression of memory. By and large, these ideas have been disconfirmed, in many cases by those who advanced them.

Some prima facie evidence of hippocampal involvement in mnemonic processes in animals has been obtained in studies of unit activity during learning. In these experiments, the animals learn one thing so that behavior and unit activity enter characteristic modes. The initial learning is then modified. Typically, the response mode of the majority of units in the hippocampus and related systems does not change until after the behavioral response has changed. For example, as a result of pseudoconditioning, both the animal and the hippocampal units sampled cease to respond to stimulus. When that stimulus is then paired with a US, behavioral conditioning occurs long before the majority of hippocampal units respond (Hirsh, 1973). The lag between the appearance of behavioral conditioning and conditioning-induced unit responses increases as the amount of prior habituation increases.

Three recently reported studies of discrimination reversal present confirmatory evidence. Units in the hippocampus and related structures respond differentially to a $\mathrm{CS}+$ and a $\mathrm{CS}-$, as does the animal. When the roles of stimuli serving as the $\mathrm{CS}+$ and the CS - are reversed, the behavioral responses to the stimuli are switched prior to those of the vast majority 
of hippocampal units (Disterhoft \& Segal, 1978; Gabriel, Miller, \& Saltwick, 1977; Deadwyler, Note 1). ${ }^{1}$ During such lags, the units in question are divorced from processes responsible for ongoing performance. Rather, they are coupled to past experience under the influence of a mnemonic process.

Present literature on human memory contains a fairly broad variety of distinctions between types of memory. In fact, human amnesics, including those with confirmed hippocampal lesions, learn normally in some paradigms but not in others (Sidman, Stoddard, \& Mohr, 1968; Warrington \& Weiskrantz, 1971, 1974). Thus, it is reasonable to ask which kinds of memory involve hippocampal function and which do not.

\section{Conditional Operations}

The answer I propose is that the hippocampus is involved in mnemonic processes incorporating conditional operation and not involved in those not utilizing conditional operations. Conditional operations allow two highly similar situations to be treated quite differently. Conditional probabilities are an example. The statement that given $Y_{1}, p_{a}=.5$ is in no way a contradiction of the one that given $\mathrm{Y}_{2}, \mathrm{p}_{\mathrm{a}}=.02$, despite the fact that $p_{a}$ is the same entity in both cases.

Hippocampally ablated animals do not behave conditionally in learning situations in which normal animals are quite capable of conditional operations. When conditional operations are not especially advantageous, hippocampally ablated animals learn at normal rates (Hirsh, 1974).

A recent series of experiments in our laboratory have modelled conditional relationships by requiring rats to learn to behave differentially in the same maze in order to obtain food and water. Food is placed in one arm of a T-maze and water is placed in the other. During odd-numbered sessions, animals deprived of one of the two incentives, for example, water, are allowed to choose between the end boxes in three widely separated trials. Between trials, the remaining incentive, for example, food, is freely available in the home cage between trials. During even-numbered sessions, the animals are deprived of the other incentive, food in our example, and also allowed three widely separated opportunities to choose between the end boxes. The remaining incentive is freely available in the home cage between these trials. The external environment is kept constant across deprivation conditions. Maximally adaptive behavior thus requires opposing behavior in the same external environment.

If the motivational states are operating conditionally upon the external stimuli, learning under one deprivation condition should be independent of that in the other deprivation condition. If the influences of the motivational conditions and the external stimuli over behavior are simply added together, there will be quite a bit of interference between the learning in the two conditions. Thus, the presence or absence of condi- tional operations should determine the style of learning. In addition, learning in the absence of conditional operations can be expected to be slower.

The style of learning was assessed by plotting, on the same axes, performance levels in obtaining food and in obtaining water as a function of trials. A separate graph was plotted for each individual animal so that stylistic features of the learning process would not be masked by the fact that at any given moment performance levels differed between animals within a group.

Three separate disruptions of hippocampal function have been found to produce a nonconditional style of learning: transection of all fornix fibers (Hirsh, Leber \& Gillman, 1978), destruction of dentate granule cells and their mossy fibers by means of irradiation delivered neonatally (Hirsh, Holt, \& Mosseri, 1978), and transection of only those fornix fibers bound for the anterior thalamus (Hirsh, Davis, \& Holt, 1979). The results of these three separate disruptions are typified by those presented in Figure 1.

Even at the outset of learning, the behavior of the control animals in obtaining food is independent of, that is, conditionally different from, their behavior in obtaining water. It is fair to say that while control animals first learned to obtain one incentive, their performance in obtaining the other incentive remained at chance levels. Later learning did not interfere with the initially established learning. Independence was quantitatively assessed by computing correlation coefficients for the two performance levels during the first trials for each animal. The median correlation coefficients for the control groups in the three experiments ranged between $r=-.33$ and $r=-.45$.

The experimental groups behaved as if they were confronting a single problem, responding similarly in both deprivation conditions. At the outset of learning, they tended to make just one response in both conditions, so one performance level was high while the other remained low. Even as this stereotypy disappeared, there was still little differentiation between the conditions. The probability of the occurrence of a given response did not vary across changes in deprivation conditions. Thus, as the initially low performance level improved, the initially high performance declined. The median correlation coefficients for the experimental groups ranged between $r=-.80$ and $\mathrm{r}=-.91$.

Such results cannot be attributed to response perseveration. Note that in Figure 1B the correlation between the two curves as they cross is strongly negative, even though this animal is alternating choices within sessions as frequently as did the controls at the outset of learning.

The similarity of the behavior of the experimental animals across deprivation conditions indicates that the common cues, for example the external stimuli, were exercising the same influence in both of the 
CONTROL No.21

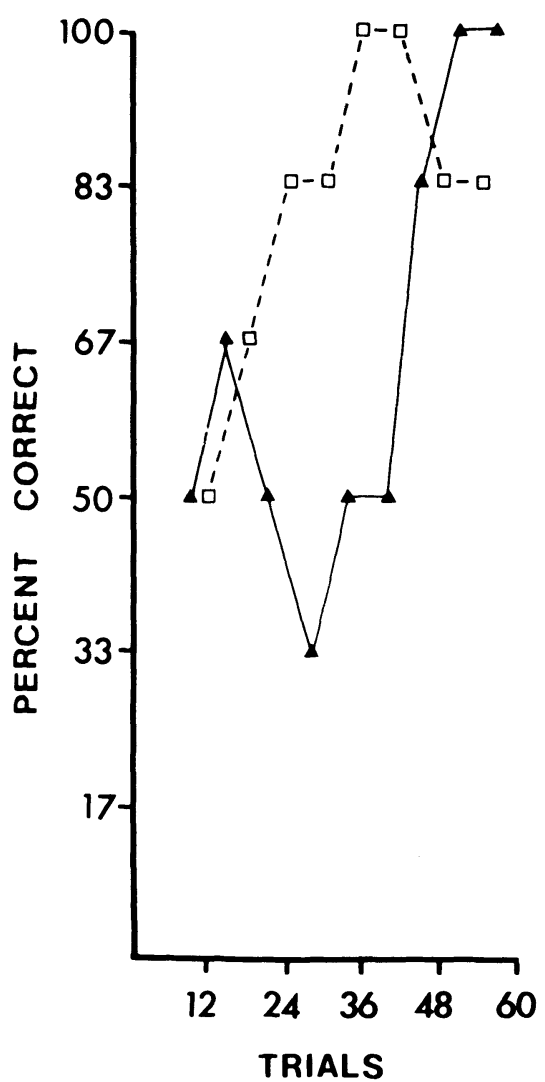

IRRADIATED No.4

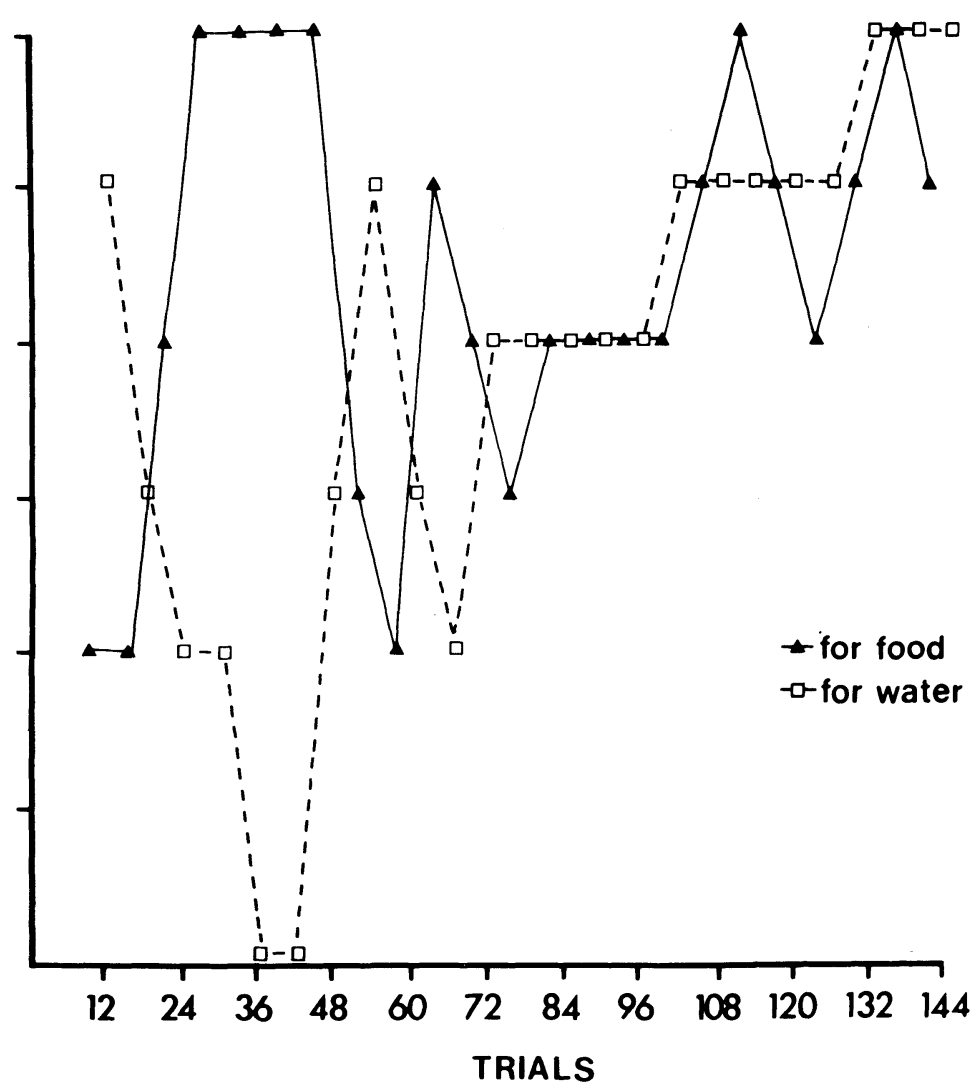

Figure 1. Performance in obtaining food and in obtaining water as a function of trials for a typical control animal and one in which dentate granule cells were destroyed by neonatal irradiation. Each of the two individual animals required the median number of trials to reach criterion in their respective groups. Each data point represents performance during a block of six trials. Each successive block overlaps three trials of the preceding one.

conditions. There is evidence that this invariant influence of the common cues declines only gradually. In the experiment assessing the effects of transecting fornicothalamic fibers, correlation coefficients were computed for performance levels in the last 36 trials prior to the beginning of each animal's criterion run. The median value for the controls was $r=-.12$, while that for the experimental animals was $r=-.45$, a value similar to those for the behavior of the controls at the outset of learning.

In the experiments just described, hippocampal disruption leads to a loss of conditional operations by motivational states upon a set of spatially defined choices during learning. A loss of a general ability to acquire and apply conditional relations can explain a wide variety of reported effects of hippocampal lesions. Such an explanation is not in direct opposition to many of the others advanced in this issue. Rather, it is the embodiment of a theme that is common to many of them.

Hippocampal ablation apparently affects a wide variety of conditional behaviors. Although hippo- campectomized animals have no trouble learning a simultaneous black vs. white discrimination, they are decidedly deficient in learning a conditional discrimination between these two cues (Kimble, 1963). This situation may be cast as one in which learning requires that brightness cues operate conditionally upon a spatially defined set of choices.

Truly sequential behavior, such as that incisively analyzed by Olton and his colleagues (Olton \& Samuelson, 1976), incorporates a conditional relation. The animal is confronted with a number of possibilities, all of which have been equally experienced over the long term as adaptive. The appropriateness of the options is thus not dependent on experience of factors intrinsic to them. Instead, it rests upon the choices already made, information stored in working memory for the appropriate duration. The contents of working memory operate conditionally upon the set of possibilities to produce sequential behavior. Hippocampal disability clearly reduces the ability of working memories of spatially defined choices to conditionally operate upon further spatially defined choices (Olton, Walter, \& 
Gage, 1978). It also affects sequential behavior that is not obviously spatial. For example, if tones alternately signal inescapable shock and no-shock within a small box, normal rats will learn to react differentially, as measured by cardiac rhythms, on alternate trials. Hippocampally ablated animals will show substantially the same learned behavior on all trials (Caul, Jarrard, Miller, \& Korn, 1969).

Most of the early work on the behavioral effects of hippocampal ablation in animals can be described as demonstrating deficiencies in situations requiring modification of already established learning. For example, hippocampally ablated animals have no trouble in acquiring simultaneous discriminations. However, they are much slower than controls in reversing those discriminations once they have been established. This is true for brightness (Silveira \& Kimble, 1968), shape (Douglas \& Pribram, 1966), orientation (Olton, Becker, Handelmann, \& Mitchell, 1980), and tactile (Teitelbaum, 1964; Webster \& Voneida, 1964) discriminations, as well as those that are obviously spatial. It should be noted that many of the other paradigms requiring modification of prior learning, which are diagnostic for hippocampal dysfunction, are not obviously spatial either.

Modification of previously established learning may be cast as requiring conditional operations by assuming that the initially acquired information is retained rather than erased. Such phenomena as the formation of reversal and extinction sets indicate that this assumption is valid. The retained and subsequent learning would comprise a set of possibilities that would be operated upon conditionally by a historical marker. For instance, the initially established learning would be considered true in the past, while the subsequent learning would be considered true in the present. Such markers could be constructed only from information in long-term storage and would reside there.

Hippocampal dysfunction would incapacitate the formation and utilization of conditional relations between the historical markers and the memories describing the discriminanda. The discriminanda would exercise an influence upon behavior in their own right, unrelated to historical markers. Previous learning would interfere with subsequent learning. In the absence of a referral process, the only way to eliminate interference is by erasing the initial learning. Evidence that such erasure occurs in hippocampally ablated animals is provided by the observation that they are incapable of forming reversal (Kimble \& Kimble, 1970) or recovery (Schmaltz \& Theios, 1972) sets despite the fact that they do eventually achieve discrimination reversal and demonstrate complete extinction.

Episodic memories make the fullest possible use of historical markers as conditional operators. Episodic memories are essentially descriptive history, for example the events that happened yesterday, rather than representations of meaning or semantic memory. Most histories comprise rather commonplace events which can be expected to appear in many other memories, both episodic and semantic, all of which would be activated in a process that did not refer particular events to a temporal marker. The information that the events occurred together in time would be lost. The historical quality of remembered information can be retained by referring the events to the specific time and place in which they occurred, a process Kesner (1980) holds to be a true consequence of normal hippocampal function. Such references would, in effect, be statements that the remembered information is applicable only under the most limited conditions.

\section{Retrieval and Cognition}

Conditional determination of which stored information will control behavior is a form of retrieval. By retrieval, I mean the selection from a larger set of potentially relevant memories of a single or smaller set of memories of even greater relevance and thus greater potential for controlling behavior in the situation facing the animal. Defined in this manner, retrieval is something considerably more than is implied in the commonplace conceptual sequence "acquisition, storage, and retrieval." For starters, retrieval is not necessarily the antithesis of acquisition and/or encoding. In some conceptions, acquisition and retrieval clearly occur simultaneously. For example, acquisition of the solution to a particular problem can consist of hypothesis testing, placing pieces of information acquired in solving other problems in tentative control of behavior to test their utility. Such casting or encoding of a solution to a present problem in terms of what is already known requires retrieval. Moreover, simply entering information into a store is of little value. The information must be filed in such a way as to be accessible under appropriate conditions. Filing and retrieval of already stored information are in many ways symmetric. It is quite conceivable that they incorporate many of the same logical operations and thus depend, in part, upon the same brain structures.

More important, cognitive processes can be only as rich as retrieval mechanisms allow. Theories that eschew all forms of cognition, that is, elementary associative ones, are constructed so as to make the issue of retrieval trivial by assuming that that process is nothing more than the identification of the stimulus. This assumption is tacitly made by the proponents of elementary associative theories when they hold that learning is the linking of the neural entity activated by one event with that activated by another event. The nature of this link is such that the activation of one neural representation activates the other, without any other possible recourse. The neural representations comprising the memory are not merely related; they 
are fused into a single entity. Such fusion makes incorporation of the same event into more than one memory unacceptable because the occurrence of that event would activate conflicting memories. Associative theories resolve this difficulty by eliminating the possibility of the same event being incorporated in two or more memories. For instance, in S-R theories, reinforcement following an $S_{1}-R_{1}$ strengthens the associative bond, while nonreinforcement following an $S_{1}-R_{2}$ results in a weakened bond, so that optimal training procedures result in the presence of just one memory incorporating the $S_{1}$. In such circumstances, there is no more information about a situation present within the nervous system than is apparent in behavior at that instance, hence the appellation "behaviorism." Thus, the occurrence of the stimulus is sufficient to ensure the activation of the appropriate memory. There is no need for any particularly selective operations.

It should be noted that, according to elementary forms of associative theories, learning is the induction of stereotype. Such a process is adaptive only to the extent that the behavior of the world can be resolved into stereotypes.

In contrast, cognitive theories hold that animals may know more than one thing at a time about a stimulus and that one of these pieces of knowledge is selected to control behavior in a given instant. For example, in place-learning experiments, a learned animal placed in one apex of a triangular maze will directly approach whichever of the other two corners in which it was just shown food. The cognitive interpretation is that the animal has learned that each corner of the maze is connected to the other two and that the presence of the incentive in some manner marks which of the acquired relations is to be applied to controlling behavior.

To differentiate between memories incorporating the same stimuli, it is necessary for the various components of the memory to remain distinct even while they are related. This may be done relatively simply by casting the memories as and-gates activated by a specific combination of stimuli. For instance, in the experiment in which food and water are in opposite arms of the maze, one memory would be activated only when the animal was hungry and in the maze, while the other would be activated only when the animal was thirsty and in the maze. Such unique combinations are termed "configurations" by associative theorists, who, for the most part, find the idea quite congenial (Wickelgren, 1979).

I am not sure that this position is entirely valid. The nodes in the neural network representing or detecting a particular combination receive input from nodes representing the individual components. To ensure flexibility, nodes detecting the individual components are allowed to send inputs to detectors for other combinations and to have meaning in their own right. The latter case reintroduces the possibility of conflict, if activation of memories is solely a function of events in the sensorium.

To illustrate, consider a case in which light alone signals one response, a tone alone signals that a different response is in order, and light and tone together signal yet a third response. When light and tone are presented in combination, the node detecting the light and the one detecting the tone will be activated along with the one detecting the combination, resulting in some tendency for each of the three responses to occur. Moreover, the primary nodes will be activated prior to the combinational one. Thus, a postulate of mutual inhibition between nodes is only a partial solution to the problem of competition.

\section{Conditional Retrieval and Dimensions}

Although conditional retrieval incorporates andgates, it is something more than a simple conjoint operation. In a simple conjoint relation, the various inputs play equivalent roles. The arrangement is, in essence, a partnership. In contrast, conditional retrieval postulates two functionally different kinds of cues, the categorical and local operators, which differ in status on the basis of scope.

Based on relatively global concerns, the categorical operator defines the set of potentially relevant memories. This set of memories, which in some sense is already active, is further constrained by whatever local cue is currently operating. For example, in the experiment in which obtaining food and water required opposing responses, the state of hunger may be cast as one of a set of categorical operators. When it operates, the set of relevant memories is defined as one describing the acquisition of food. The maze cues, which are present only some of the time that the animal is hungry, may be cast as one member of a set of possible local operators. Their presence further constrains the set of relevant memories to that of the goalbox containing food during the hunger trials. As in other varieties of sorting trees, the categorical operator limits the sphere of operation of the local cues rather than operating conjointly with them. Within this functional differentiation, there is a potential for hierarchical control.

However, note that the categorical and local operators are defined in terms of the roles in respect to each other, rather than in terms of their source or content. A given cue can serve as the local operator vis-à-vis one serving as a categorical operator and as a categorical cue for an even more local operator in the same instance of behavior. It is even conceivable that the roles of a given pair of cues may be reversed. For example, the maze containing food and water may be cast as the categorical operator defining the set of potentially relevant memories as all of those describing the parts of the maze. The state of hunger, which operates only some of the time that the animal is in 
the maze, may be cast as the local operator constraining the set of memories to the goalbox containing food. While the outcome in this example is the same as that in the previous one, the two cases are not equivalent, because the larger sets of potentially relevant memories were quite different.

Although distinct categorical and local operators may not be necessary for retrieval, they are required to construct maps and other dimensional characterizations. Categories, as the term is currently used, are distinct from dimensions. Categories are sets of objects or events having common properties, for example, buildings, personalities, virtues. They identify the constancy across the members of the set by disregarding the variation between them. Because associative processes work in precisely this manner, they are well able to form inclusion sets.

Dimensions, in contrast, systematically characterize variation across a set of events or objects, for example, space, intelligence, or honor. To identify or construct a dimension distinct from others, it is necessary to compare a set of objects or events that are posited to be otherwise equivalent. For instance, the ideas of area or shape as distinct dimensions cannot be achieved by comparing a large triangle with a small circle because there is nothing to prevent the area of one being related to the shape of the other. To construct or identify a dimension, it is thus necessary to identify both constancy and variation and to relate them, which is precisely what a conditional relation does.

In constructing a dimension, the categorical operator is available to define the set of objects or events tentatively assumed to be equivalent in all ways but one, for example, circles. The identity of each individual of the categorical set may function as a local operator, thereby yielding a value along a coherent dimension. For example, one circle in a set of them may have an area of $16 \mathrm{sq}$ in., while another will have one of $4 \mathrm{sq}$ in. The coherence of the resulting set of values may be assessed by comparing them to those derived from some other categorical set, for example, a set of triangles, assumed to vary in the same way.

\section{Conditional Retrieval and Maps of all Kinds}

Maps, as one form of expression of dimensional relations, rest upon conditional operations. O'Keefe and Nadel (1978), in my opinion, are correct in describing the behavior of hippocampally ablated animals as bound to one particular route in the absence of a capacity to generate maps. Their distinction between routes and maps is a more graphic and lucid expression of the one I made (Hirsh, 1974) between processes in which information is stored on and off the control line, the control line being defined as the series of events initiated by the immediately observable stimulus and terminating in the immediately observable response.
A map contains potentially contradictory information in that the same stimulus is related to many others. Thus, selection of one particular stored relation to guide behavior must depend upon something other than the occurrence of the immediate stimulus. The remembered items available for such selection are, by definition, stored off the control line. In a conditional retrieval process, the categorical operator would serve as the factor supplementing the immediate stimulus. Route following and processes guided by information stored upon the control line are isomorphic in that both hold that behavior is entirely determined by the occurrence of the stimulus. In consequence, each posits that learning is a form of stereotypy. As such, both are highly reminiscent of elementary associative theories.

Furthermore, I agree with O'Keefe and Nadel that an absence of expectations is the appropriate way to account for such effects of hippocampal ablation as more rapid learning in a two-way avoidance situation, reduction in the consequences of changing amount of incentive, and reduced incidence of frustration. Both they and I predicate the occurrence of expectations upon the existence of what can be termed maps and assume that the hippocampus is necessary to generate and utilize them. The sole difference is that O'Keefe and Nadel hold that the logical operations occurring within the hippocampus are applied only to information about space in the literal sense, whereas I hold that they are applied to problem spaces in the sense of analytic geometry, of all kinds.

The utility of the narrow definition of "map" in characterizing hippocampal function is limited. Much of the data on hippocampal unit activity were generated in classical conditioning paradigms, as were some of those on the effects of hippocampal ablation. Some of the latter are clearly analogous to those observed in more instrumental formats. To consider a knowledge of place per se as necessary or expeditious for performance in classical conditioning is something of a contortionist's trick. O'Keefe and Nadel recognize the limit of the narrow definition of map in dealing with the effects of hippocampal lesions in humans. They broaden the definition of map almost insouciantly to account for the generalized amnesia observed in humans, thereby positing an unsubstantiated evolutionary discontinuity between humans and other mammals.

Employment of the broad definition of "map," in contrast, allows the data on hippocampal function to be applied to fundamental questions in both the psychology of learning and the development of cognitive processes. Moreover, it allows present knowledge of the integrative processes within the hippocampus to be used as a starting point in unraveling the neuronal circuits responsible for carrying out the logical operations underlying mnemonic and cognitive processes in general.

The history of the psychology of learning is one of the tension between S-R theories, as exemplified by 
Hull's (1943) book and the more cognitive theories of Tolman, which I would epitomize in the postulations of cognitive maps (Tolman, 1948) and purposive behavior (Tolman, 1932). O'Keefe and Nadel (1978) and I claim that hippocampally ablated animals necessarily behave in ways predicted by elementary S-R theories in situations in which normal animals are capable of constructing maps. Abe Black realized that if this claim was correct, then both map-constructing and elementary associative systems must, in some manner, be present in the brain. The paper by Black and Dalrymple (Note 2) in this issue confirms that deduction in the course of elucidating the factors determining whether the elementary associative or mapping system operates in a spatial task. A generalization of these findings to other kinds of tasks would settle the central controversy in the psychology of learning in favor of both positions.

Such a solution is anything but parsimonious, which, from a technical point of view, is a serious drawback. Such a theory can make no definitive predictions in many experimental situations and at the same time account for almost anything after the fact as some of the discussions during this meeting have illustrated too well.

At the same time, the presence of both of the simple associative and the cognitive systems is of considerable adaptive and developmental value. The strength and weaknesses of the associative and cognitive processes are mutually complementary. The associative system must have been present all along if it is free to express itself following hippocampal ablation. The use of maps allows behavior to be highly flexible, to undergo radical shifts in direction as a consequence of relatively subtle conditional changes. However, as the discussions of hypothesis-testing and dimension formation attest, a cognitive strategy requires a certain amount of information as to what aspects of the sensory input are of potential relevance, before learning can begin.

In contrast, the properties that make associative processes rigid are the same ones that allow them to operate with almost no prerequisites. An associative strategy is akin to signal averaging. No supposition whatsoever is made about the sensory input. Instead, the ability of every element comprising the input to act as a stimulus is subjected to the influence of reinforcement or nonreinforcement on every trial. The influences accumulate in an additive fashion. As a result, the stimulus elements correlated with reinforcement are incorporated into strong associations. Elements of the sensory input not correlated with reinforcement enter into rather nondescript associations as the effects of reinforcement and nonreinforcement cancel each other. Thus, an associative strategy is to be recommended to young and inexperienced organisms, not only as a means of modifying behavior, but also as a means of isolating constellations of sen- sory input having stimulus value and thereby acquiring the categorical information prerequisite to map construction and utilization.

My attention was directed toward developmental processes by Rose (Note 3), who pointed out that the characterization of the difference between hippocampally ablated and normal animals by O'Keefe, Nadel, and myself in terms of map and dimension construction and utilization is very much analogous to the way developmental psychologists characterize the difference between 4- and 7-year-old children (Kendler \& Kendler, 1959; White, 1965). In this light, the finding that roughly $90 \%$ of dentate granule cells differentiate and connect with the pyramidal cells of Ammon's horn between 8 and 30 days after birth (Altman \& Das, 1965, 1966) in the rat is highly suggestive in that, by certain criteria, this period is roughly comparable to ages 4-7 in humans.

Another broad implication of studies of hippocampal function in producing behavior relates to the question of how neuronal circuits carry out the logical operations comprising mnemonic and cognitive processes. Findings on the lamellar organization of the hippocampus indicate that the same basic kinds of circuits are repeated throughout it (Andersen, Bliss, \& Skrede, 1971; Blackstad, Brink, Hem, \& Jeune, 1970). The laminar organization within each lamella permits the attribution of electrophysiological signals to particular morphologically defined elements. Thus, the wherewithal for theoretical syntheses about the logical operations carried out by circuits of neurons is present in the hippocampus to an extent matched by few other forebrain structures. The hippocampus, then, is the ideal place to begin unraveling the neuronal circuitry carrying out the logical operations comprising psychological processes. If the psychological processes in which the hippocampus is involved are narrowly defined in terms of geographic location, then the scope of this opportunity will be severely curtailed, because the structure and probably much of the neurophysiology of the hippocampus are unique. Generalization to the operations comprising other nonspatial processes based on hippocampal circuits would thus be highly problematical.

My position on the utility of characterizing the function of the hippocampus in terms of construction and utilization of maps of all kinds can best be summed up by an anecdote. It was the Day of Atonement. Each member of the congregation was fervently praying to be forgiven for his sins, when one man began to mutter at the Lord. In answer to the rabbi's inquiries, the man replied that it had occurred to him that the harm his sins had wrought was trivial alongside the harm caused by famines, plagues, or earthquakes. Thus, he had offered to forgive God if God would forgive him. The rabbi was furious. "With an argument like that," he said, "you could have forced Him to forgive everybody; why did you stop with 
yourself?" With a process as powerful as conditional retrieval in constructing and utilizing cognitive maps, we should not stop its application at place learning.

\section{REFERENCE NOTES}

1. Deadwyler, S. Personal communication, April 1979.

2. Black, A. H., \& Dalrymple, A. J. Reasoning in the rat reconsidered. Manuscript in preparation.

3. Rose, D. Personal communication, June 1975.

\section{REFERENCES}

Altman, J., \& Das, G. B. Autoradiographic and histological evidence of postnatal hippocampal neurogenesis in rats. Journal of Comparative Neurology, 1965, 124, 319-335.

Altman, J., \& Das, G. B. Autoradiographic and histological studies of postnatal neurogenesis I. A longitudinal investigation of kinetics, migration, and transformation of cells incorporating tritiated thymidine in neonate rats with special reference to postnatal neurogenesis in some brain regions. Journal of Comparative Neurology, 1966, 126, 337-390.

Andersen, P., Bliss, T. V. P., \& Skrede, K. K. Lamellar organization of hippocampal excitatory pathways. Experimental Brain Research, 1971, 13, 222-238.

Blackstad, T. W., Brink, W., Hem, D. V., \& Jeune, B. Distribution of hippocampal mossy fibers in the rat. An experimental study with silver-impregnation methods. Journal of Comparative Neurology, 1970, 138, 433-450.

Caul, W. F., Jarrard, L. E., Miller, R. E., \& Korn, J. H. Effects of hippocampal lesions on heart rate in aversive conditioning. Physiology \& Behavior, 1969, 4, 917-922.

Disterhoft, J. F., \& Segal, M. Neuron activity in rat hippocampus and motor cortex during discrimination reversal. Brain Research Bulletin, 1978, 3, 583-588.

Douglas, R. J., \& Pribram, K. H. Learning and limbic lesions. Neuropsychologia, 1966, 4, 197-220.

Gabriel, M., Miller, J. D., \& Saltwick, S. E. Unit activity in cingulate cortex and anteroventral thalamus of the rabbit during differential conditioning and reversal. Journal of Comparative and Physiological Psychology, 1977, 91, 423-433.

Hirsh, R. Previous stimulus experience delays conditioning induced changes in hippocampal unit responses in rats. Journal of Comparative and Physiological Psychology, 1973, 83, 337-345.

Hirsh, R. The hippocampus and contextual retrieval of information from memory: A theory. Behavioral Biology, 1974, 12, 421-444.

Hirsh, R., Davis, R., \& Holt, L. Fornico-thalamic fibers, motivational states and contextual retrieval. Experimental Neurology, 1979, 65, 373-390.

Hirsh, R., Holt, L., \& Mosseri, A. Hippocampal mossy fibers, motivational states and contextual retrieval. Experimental Neurology, 1978, 62, 68-79.

Hirsh, R., Leber, B., \& Gillman, K. Fornix fibers and motivational states as controllers of behavior: A study stimulated by the contextual retrieval theory. Behavioral Biology, 1978, 22, 463-475.

Hull, C. L. Principles of behavior. New York: Appleton-CenturyCrofts, 1943.

Jarrard, L. E. The hippocampus and motivation. Psychological Bulletin, 1973, 79, 1-12.

Kendler, T. S., \& Kendler, H. H. Reversal and nonreversal shifts in kindergarten children. Journal of Experimental Psychology, 1959, 58, 56-60.

KESNER, R. An attribute analysis of memory: The role of the hippocampus. Physiological Psychology, 1980, 8, 189-197.

KImBLE, D. P. The effects of bilateral hippocampal lesions in rats. Journal of Comparative and Physiological Psychology, 1963, 56, 273-283.

Kimble, D. P., \& Kimble, P. J. The effect of hippocampal lesions on extinction and "hypothesis" behavior in rats. Physiology \& Behavior, 1970, 5, 735-738.

O'KeEFE, J., \& NADEL, L. The hippocampus as a cognitive map. New York: Oxford University Press, 1978.

Olton, D. S., Becker, J. T., \& Handelmann, G. E. Hippocampal function: Working memory or cognitive mapping? Physiological Psychology, 1980, 8, 239-246.

Olton, D. S., \& Samuelson, R. J. Remembrance of places passed. Spatial memory in rats. Journal of Experimental Psychology: Animal Behavior Processes, 1976, 2, 97-116.

Olton, D. S., Walter, J. A., \& Gage, F. H. Hippocampal connections and spatial discrimination. Brain Research, 1978, 139, 295-308.

Penfield, W., \& Milner, B. Memory deficit produced by bilateral lesions in the hippocampal zone. Archives of Neurological Psychiatry, 1958, 79, 475-497.

Schmaltz, L. W., \& Theios, J. Acquisition and extinction of a classically conditioned response in hippocampectomized rabbits (Oryctolagus cuniculus). Journal of Comparative and Physiological Psychology, 1972, 79, 328-334.

Sidman, M., Stoddard, L. T., \& Монr, J. P. Some additional quantitative observations of immediate memory in a patient with bilateral hippocampal lesions. Neuropsychology, 1968, 6, 245-254.

Silveira, J. M., \& Kimble, D. P. Brightness discrimination and reversal in hippocampally lesioned rats. Physiology \& Behavior, $1968,3,625-630$

Teitelbaum, H. A. A comparison of orbito-frontal and hippocampal lesions upon discrimination learning and reversal in the cat. Experimental Neurology, 1964, 90, 452-462.

Tolman, E. C. Purposive behavior in animals and men. New York: Century, 1932.

Tolman, E. C. Cognitive maps in rats and men. Psychological Review, 1948, 56, 144-155.

Warrington, E. K., \& Weiskrantz, L. Organizational aspects of memory in amnesic patients. Neuropsychologia, 1971, 9 , 67-73.

Warrington, E. K., \& Weiskrantz, L. The effect of prior learning on subsequent retention of amnesic patients. Neuropsychologia, 1974, 12, 419-428.

Webster, D. B., \& Voneida, T. J. Learning deficits following hippocampal lesions in split brain cats. Experimental Neurology, 1964, 10, 170-182.

White, S. H. Evidence for a hierarchical arrangement of learning processes. In L. P. Lipsitt \& C. C. Spiker (Eds.), Advances in child behavior and development (Vol. 2). New York: Academic Press, 1965.

Wickelgren, W. A. Chunking and consolidation. A theoretical synthesis of semantic network, configuring in conditioning, S-R versus cognitive learning, normal forgetting, the amnesic syndrome and the hippocampal arousal system. Psychological Review, 1979, 86, 44-50.

\section{NOTE}

1. These findings make for a rather interesting contradiction when juxtaposed with findings that hippocampal ablation results in slower discrimination reversal. 